\title{
PETER - A Pan-European Training, research and Education network on Electromagnetic Risk Management
}

\author{
Davy Pissoort ${ }^{1}$
}

KU Leuven, Bruges Campus, BE

\begin{abstract}
Sophisticated electronic technologies are increasingly used in missionand safety-critical systems where electromagnetic interference (EMI) can result in substantial risks to people and the environment. Currently, EMI engineering follows a rule- based approach, which is unable to cope with complex modern situations. With this rules-based approach, during the design stage, guidelines are used, which result in the application of a set of mitigation techniques, which are verified in the finished product against standards. This rule-based approach is costly, but with no guarantee of the required performance. This is particularly so for sensitive medical applications or the fully autonomous systems that are becoming ever-more common in our society. What we need is a risk-based approach, which is what PETER ${ }^{2}$, the Pan-European Training, Research \& Education Network on Electromagnetic Risk Management, will provide. PETER is training 15 young engineers in topics related to the development of high-tech systems that maintain reliability and safety over their full life-cycle, despite complex EMI, such as in hospitals or transport systems. This is achieved using best practices and state-of-the-art EM engineering, reliability engineering, functional safety, risk management and system engineering, to create the riskbased EMC approach.
\end{abstract}

\section{Introduction}

Electromagnetic interference (EMI), familiar to most people as the buzzing sound from a loudspeaker when a mobile-phone call is received, is becoming much more than just an annoyance. High-tech electronic equipment in our cars, homes, workplaces and hospitals is increasingly vulnerable to EMI. As a recent example:

\footnotetext{
${ }^{1}$ PETER project coordinator, professor at KU Leuven Bruges Campus. E-mail: davy.pissoort@,kuleuven.be

${ }^{2}$ https://etn-peter.eu

(c) KU Leuven 2021.

Published by the Safety-Critical Systems Club. All Rights Reserved
} 
in May 2018, one of Mobileye's prototypes of autonomous cars went straight through a red light about a quarter of mile from the company's garage after an otherwise uneventful ride. And, of course, this happened at well-attended press release. Mobileye's Chief Executive Officer Amnon Shashua said wireless transmitters on cameras used by the television crew created electromagnetic interference, which disrupted signals from a transponder on the traffic light ${ }^{3}$.

What all this means is that as technology advances and we are able to produce machines like autonomous vehicles, which are likely safer than those driven by humans, the problem of EMI-related risks does not simply go away. Instead, we need to shift our focus away from the relatively visible risks associated with conventional devices, to understanding how the enormously complex systems that we are constructing around us are also susceptible to risk, but a different kind of risk that is associated with invisible EMI. So, as advances in medicine lead to greater survival rates after surgery, for example, and to fewer post-operational problems, the number of reported incidents in hospitals relating to EMI is also steadily increasingly, as a search in the US MAUDE database reveals (Fig. 1). Paradoxically, at the same time as we improve the quality of lives, reducing many familiar risks and allowing people to enjoy a lot of things that were not available to them less than a generation ago, we expose ourselves to other dangers like EMI that require a very different approach to managing risk in complex scenarios.

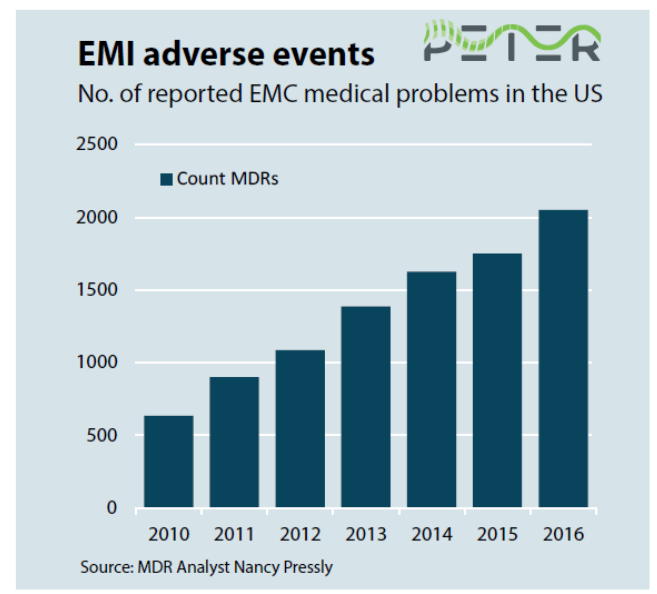

Figure 1: Increase in the number of electromagnetic interference medical events in the US

To get an idea of how the problem of EMI could develop as electronic equipment becomes more complex and we become increasingly reliant on its dependability, let's expand on a couple of very plausible scenarios. Imagine an explosion at an aging chemical plant. Firefighters arrive quickly, bringing the usual hoses and

\footnotetext{
3 https://www.bloomberg.com/news/articles/2018-05-21/why-intel-s-mobileye-blew-a-red-lightwhile-showing-off-its-ride (accessed on Nov 30 2020)
} 
ladders, but also the latest high-tech camera drone to assess the extent of the damage and identify the access paths through the site. While leading the firefighters through the smouldering remains of the plant, the drone flies too close to the plant's old wireless-communication system and is exposed to a higher field strength than it is designed and tested for. The remote operator of the drone sees the system crash, and with it the drone. Fortunately, no one is hurt that day, but the firefighters have a lucky escape. In the second example, a big-city hospital is involved in a remote surgical operation. A woman is having a craniectomy, with the knife being wielded by a robot, controlled by a surgeon sitting in a room more than $2000 \mathrm{~km}$ away. This is one of the most dangerous and difficult operations, performed by only a few of world's leading specialists. During the final stages an electrosurgical procedure is required, which involves a radio-frequency electric surgery knife. Instantly, the images on the monitors become distorted, the LED status lights flicker ominously, and a human life is in danger.

\subsection{The flaws in the current "rule-based" approach}

Currently, the problem of EMI is adressed using a "rule-based" approach. What this means is that during the design phase for a piece of electronic equipment a number of guidelines/standards (e.g. the IEC 61000-x series) are used resulting in the default application of a set of mitigation techniques (filtering, shielding, cable routing, etc.). But as the examples above illustrate, such an approach has some serious flaws when it comes to modern high-tech systems and high-safety criticality applications like medical systems and remotely driven vehicles. This is because tackling the problem by applying rules leads to too many failure scenarios being overlooked and giving us false confidence when it comes to how reliable and safe a new system actually is. Therefore, in order to make sure that people's safety is not compromised in this way, the PETER consortium is developing a novel and much more robust "risk-based" approach to EMI management.

The weakness of the rule-based approach is that it has several major shortcomings. Firstly, we have no certainties when it comes to knowing whether these mitigation strategies, or rules, are really sufficient. Even if we could be sure of this, most of us know from experience that standards always lag behind technological developments and are based on economic and technical compromises. Perhaps the biggest worry is that immunity testing in electromagnetic compatibility standards only covers one EMI disturbance at a time, meaning that simultaneous EMI effects are not addressed. While such simultaneous EMI effects would easily occur in practice, e.g. someone making a phone call while at the same time touching a display. The first generating electromagnetic fields in the air and the latter possibly leading to an electrostatic discharge. Added to this we have the problem that the testing of large installations 
is limited to just the sub-systems being evaluated when they are brand new. But of course, EMI is a "whole system" property with many of the effects resulting from environmental factors like ageing, vibration, and temperature, as well as manufacturing variability or the impact of maintenance, repairs and upgrades.

\subsection{PETER's solution: a risk-based approach}

What is needed is a truly interdisciplinary - but also revolutionary - approach to this very serious problem. A safer environment based on assessing risk requires bringing together expertise from four operating key areas - electromagnetic compatibility, reliability engineering, functional safety and risk management - and the implementation of a risk-based approach. The PETER project - which started on April $1^{\text {st }} 2019$ and will run for four years - will consider the complete system over its whole lifecycle, i.e., from the earliest concept to the final decommissioning. The risk-based approach, which will eventually replace the outdated rule-based approach in high tech systems, involves 3 steps: hazard-and-risk analysis, risk reduction, verification and validation.

PETER's main goal is to train 15 early-stage researchers (ESRs) in topics related to the development of high-technology systems that maintain reliability and safety over their full life-cycle, despite these systems being subjected to severe and complex EMI threats.

\section{PETER's Consortium}

The PETER project involves an European-wide consortium of 13 Beneficiaries and six Partner Organisations from five EU countries (see Figure 2). Of the 13 Beneficiaries, six are from industry and two are industry-oriented research centres, which will give the 15 ESRs tremendous opportunities to obtain industry-relevant training.

The PETER Beneficiaries are six high-technology companies, including Barco and Melexis (BE), Nedap and RHM (NL), MIRA (UK), and Valeo (FR), two nonuniversity research institutes, WIS and FHG (GE), and 5 universities, KU Leuven (BE), LUH (GE), UTwente (NL), UoY (UK), and ESEO (FR). The consortium is completed by six Partner Organisations that include four companies, a hospital and a university. 


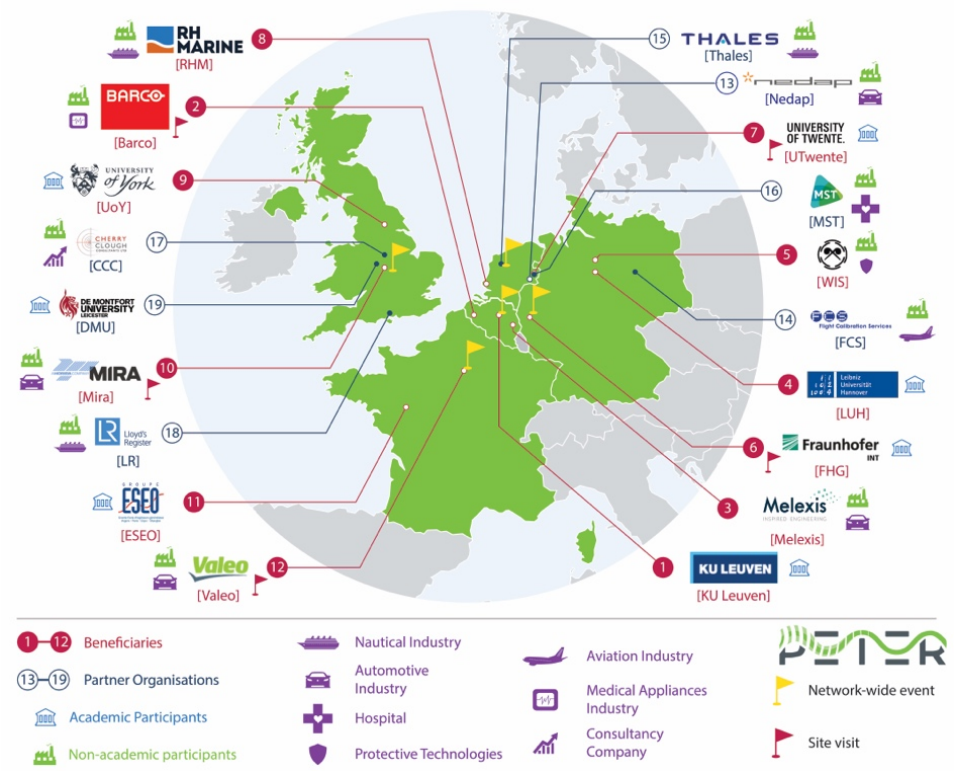

Figure 2: Map showing the locations of the PETER Beneficiaries and Partner Organisations, together with the Network-Wide Events and Site Visits

\section{PETER's Work Package Structure}

PETER has four scientific and technical (S\&T) objectives based around four S/T Work Packages (WPs), see Figure 3:

- To develop dedicated hazard-and-risk analysis techniques that identify all EMI-related risks and hazards for a system under development in its actual operating electromagnetic environment, including the possibility of malicious EMI (WP1).

- To develop effective EMI risk-reduction techniques in hardware and software, and to reduce the risks to the level where they are no longer unacceptable with respect to reliability or safety (WP2).

- To improve EMI verification-and-validation methods that represent a much broader area of the lifecycle of the system as well as of the system's actual electromagnetic environment (WP3).

- To apply a practical, industry-driven EMI risk-management methodology during four case studies, from different industrial sectors (maritime, medical, automotive, critical infrastructures) and at different design 
levels (integrated circuits, subsystems, systems and networks of systems) (WP4).

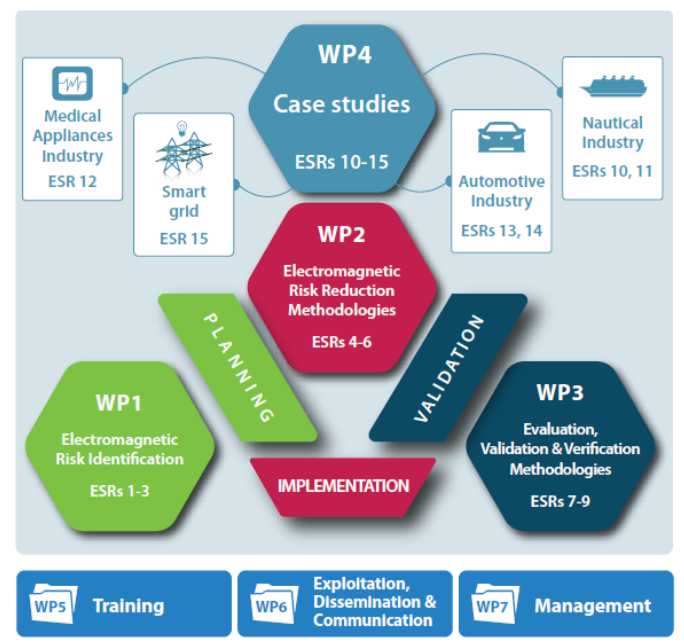

Figure 3: PETER Work Package Overview

\subsection{WP1: Electromagnetic Risk Identification}

WP1 involves three ESRs and focuses on the left part of the V-model (Figure 3) as the first step in a complete risk-based approach, i.e., the identification of all the potential EMI-related risks and their criticality. ESR1 and ESR2 take up the challenge of developing a generic electromagnetic risk-analysis methodology applicable to large and complex systems. This procedure extends the current interference descriptions of separate subsystems to a generic risk description of the whole interconnected system, e.g., from a smart machine to a critical infrastructure. It will take into account possible non-technical aspects (accessibility, regulations, etc.). ESR1 and ESR2 will be working closely on complementary projects, with ESR1 having the task of laying the theoretical fundamentals of the risk-analysis methodology, while ESR2 provides the experimental analysis and verification.

Starting from the perspective of automotive applications, ESR3 will focus on a risk-based electromagnetic engineering approach aligned with the ISO 26262 Functional Safety approach. The great challenge here is that this approach should cover safety, cybersecurity and functional performance. As such, it will be aligned with ISO 26262 functional safety concepts and the SAE Cybersecurity Guidebook, as well as integrating computational electromagnetics results within classic hazard-and-risk analysis methods (fault-tree analysis, failure-mode and effect analysis, etc.). 


\subsection{WP2: Electromagnetic Risk-Reduction Methodologies}

WP2 tackles the actual design of the mission- or safety-critical systems with three ESRs focusing on techniques and measures that effectively reduce the risks (identified with the methods of WP1) to the level that they no longer represent unacceptable reliability or safety risks.

The first of these ESRs, ESR4, is going to look at the overall risk-based EMIaware design of complex systems. Starting from the risk identification (ESRs 1-3) together with a decomposition of the electromagnetic environment into tangible levels, an EMC risk-management plan (EMI-RMP) will be established. Following system-engineering principles, this EMI-RMP will drive the design, operational use and maintenance of the system. In collaboration with the other ESRs, an EMIRMP will be made for future vehicle-to-X communication systems, complex maritime systems, medical display systems inside a hospital, and a typical smart grid.

During the design, the EMI-RMP will also identify which risk-reduction techniques should be chosen and implemented. Originally, the hardening techniques (both hardware and software) used within reliability engineering and functional safety were not developed for EMI. Hence, they will need modification and optimization to be effective for EMI, thereby also fitting EMI into the safety assessment workflow a part of the Preliminary System Safety Assessment (PSSA) process. To overcome this challenge, ESRs 5 and 6 will be working on costeffective IEC 61508 Techniques \& Measures for EMI Risk Reduction and this for hardware-based and software-based techniques and measures, respectively. Thanks to the developed techniques \& measures, a significant step-forward will be achieved in the design of high-tech medical display systems, automotive communication systems, and the smart grid.

\subsection{WP3: Evaluation, Validation and Verification Methodologies}

WP3 targets novel methodologies that allow us to evaluate, validate and verify that the applied risk-reduction techniques (WP2) have been implemented correctly and that they effectively address the identified risks (WP1) over the full lifecycle. It is crucial that the analysis takes into account the full lifecycle (environmental stresses, ageing, replacement of components during maintenance etc.). Once again, two ESRs, ESRs 7 and 8, will be working together on closely related, but complementary, research topics. While they both study simulation models and testing methods for the evaluation of electromagnetic hazards due to environmental stresses, obsolescence and/or ageing, their specific focus is different. ESR7 focuses on the level of the electronic components (integrated 
circuits), while ESR8 focuses on the system level. ESR7 will apply his/her models and methods to an automotive integrated circuit in collaboration with ESR14 (WP4). ESR8 will assist ESR5 (WP2) in the validation process for the optimized IEC 616508 techniques and measures under extreme stress.

Given the complexity of current electromagnetic environments, the way electromagnetic disturbances interact with a system will become extremely sensitive to small variations. Thus, just as for the risk assessment, statistical techniques will need to be integrated into the classic EMC testing. Therefore, ESR9 will develop a methodology for the statistical verification and validation of immunity and enclosure shielding effectiveness. Evidently, this statistical verification and validation should have a lot in common with the statistical risk analysis developed by ESRs 1 and 2. Finally, together with ESR7, the statistical verification and validation will be applied to an automotive integrated circuit (ESR14, WP4).

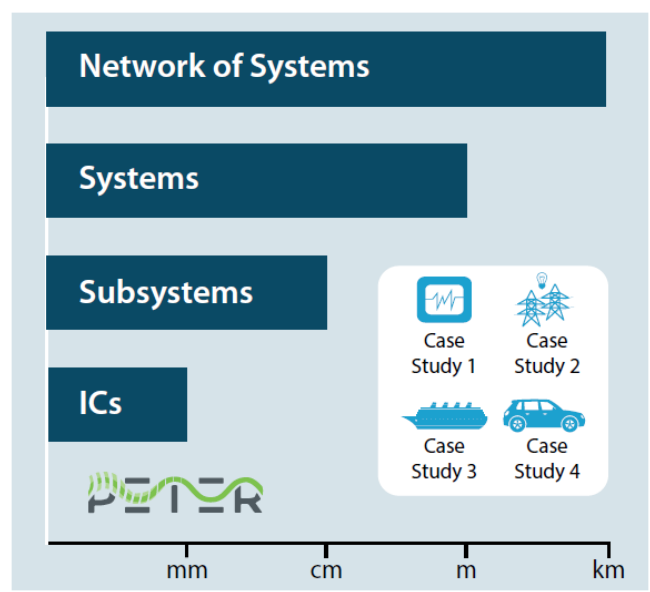

Figure 4: PETER's four case studies at 4 different design levels

\subsection{WP4: Application Case Studies}

The PETER WPs conclude with WP4, which pilots the full risk-based EMC approach, combining the previous three research tracks, thereby enabling the shift from rule-based to risk-based EMC approaches. In total, six ESRs are working on case studies for four different industrial sectors: maritime, healthcare, automotive and an energy network infrastructure in the form of a smart grid. Moreover, the case studies cover four different design levels (Fig. 4): integrated circuits (mm scale), subsystem ( $\mathrm{cm}$ scale), system (m scale) and network of systems (km scale). These case studies will not only use the results of the ESRs from WPs 1-3, but will also provide essential practical feedback for them. 
ESR 10 and 11 both focus on maritime applications. ESR10 aims at costeffective, up-to-date, maritime EMC standards that move away from the rulebased approach to a risk-based approach. ESR11 will model and reason about electromagnetic interactions in autonomous and complex vessels. ESR12 applies the risk-based EMC approach to the medical sector through the design of EMIresilient medical display systems for surgical-, diagnostic imaging-and modality applications. Both EMI and functional safety will become increasingly important within automotive applications as we shift towards self-driving, interconnected vehicles. Hence, ESRs 13 and 14 address two significant challenges related to vehicles of the future. ESR13 applies the EMI risk management to the next generation of vehicle communication devices, while ESR14 designs an automotive integrated circuit through a risk-based and EMI-aware approach. Finally, ESR15 has the task of managing in the EMI risks on the scale of the smart grid as a network of systems.

\section{Conclusions}

The goal of PETER is to achieve a risk-based approach to addressing EMI. In so doing, it will also train the researchers in this area to provice continued activities after the end of the programme. PETER proposes an interactive approach where EMI is taken into account during all the phases of a risk-management process and where this is directly applied to industry-driven application case studies. PETER will provide the first-ever high-quality interdisciplinary training in the field of risk-based EMC to 15 talented ESRs through research, taught courses and Network-Wide Events, covering scientific aspects as well as personal skills and career development.

During the PETER proposal-building phase we made sure that industry would play a major role in the project and that many aspects of the project would be industry led. Only by integrating the real-world experience of companies that succeed through their own excellence and ability to innovate and adapt can we be in a position to train the ESRs to see innovation as fundamental to their research. Every one of the 15 ESRs' $\mathrm{PhD}$ projects was assessed and then approved by theindustry Participants - Beneficiaries and Partner Organisations - in terms of its innovation potential in the European context. What we are aiming for in the PETER project is to have ESRs who see research as an opportunity to innovate, to look at problems from a new perspective, and to see the potential for marketing their ideas and knowledge.

Finally, through the participation of the ESRs and supervisors in the IEEE P1848 and IEC 61000-1-2 standardization committees, PETER aims at a strong contribution to European standardisation. 
Acknowledgments The research leading to these results has received funding from the European Union's Horizon 2020 research and innovation programme under the Marie Skłodowska-Curie Grant Agreement No 812.790 (MSCA-ETN PETER). This publication reflects only the authors' view, exempting the European Union from any liability. Project website: http://etn-peter.eu/. 